This is the authors' final version of an article originally published in Investigative Ophthalmology and Visual Science, doi:10.1167/iovs.10-6287, and is available from www.iovs.org/

\title{
Internal limiting membrane peeling versus no peeling for idiopathic full thickness macular hole: A pragmatic randomised controlled trial
}

Noemi Lois, ${ }^{1 *}$ MD, PhD; Jennifer Burr ${ }^{2}$, MD; John Norrie ${ }^{2,3}$, MSc; Luke Vale ${ }^{2,4}$, PhD; Jonathan Cook ${ }^{2}$, PhD; Alison McDonald², MSc; Charles Boachie ${ }^{2}$, MSc; Laura Ternent, ${ }^{2,4}$ MSc; and Gladys McPherson², MSc; for the Full-thickness macular hole and Internal Limiting Membrane peeling Study (FILMS) Group

*Corresponding author and reprint requests

${ }^{1}$ Ophthalmology Department, Grampian University Hospitals-NHS Trust, Aberdeen, AB25 2ZN, UK. Phone: 00-44-1224-553217, FAX: 00-44-1224-559231; Email:

noemilois@aol.com

${ }^{2}$ Health Services Research Unit, University of Aberdeen, Aberdeen, AB25 2ZD, UK.

${ }^{3}$ Robertson Centre for Biostatistics, University of Glasgow, Glasgow, G12 8QQ, UK.

${ }^{4}$ Health Economics Research Unit, University of Aberdeen, Aberdeen, AB25 2ZD, UK.

The work presented here has been submitted, as a paper, to the XXVIIth Meeting of the Club Jules Gonin, Kyoto, Japan, November 3-6, 2010.

Financial support: Chief Scientist Office of the Scottish Government Health Directorates.

No authors have any financial/conflicting interests to disclose. MS word count: 2950 


\section{Abstract}

Purpose: To determine whether internal limiting membrane (ILM) peeling is effective and cost-effective compared with no peeling for patients with idiopathic stage 2 or 3 fullthickness macular hole (FTMH).

Methods: Pragmatic, multicenter randomized controlled trial. Eligible participants from nine centres were randomized to ILM peeling or no peeling (1:1 ratio) in addition to phacovitrectomy, including detachment and removal of the posterior hyaloid and gas tamponade. The primary outcome was distance visual acuity (VA) at six months post-surgery. Secondary outcomes included hole closure, distance VA at other time points, near VA, contrast sensitivity, reading speed, re-operations, complications, resource use and participant reported health status, visual function and costs.

Results: Of 141 participants randomized in nine centres, 127 (90\%) completed six-month follow up. Non-statistically significant differences in distance visual acuity at six-months were found between groups [Mean difference 4.8, 95\% CI $(-0.3,9.8) ; \mathrm{p}=0.063$ ]. There was a statistically significantly higher rate of hole closure in the ILM peel group [56 (84\%) versus 31 (48\%)] at one month [Odds ratio (OR):6.23, 95\% CI (2.64 to 14.73); $<<0.001$ ] with fewer re-operations [8 (12\%) versus 31 (48\%)] performed by six months [OR:0.14, 95\% CI (0.05, 0.34); $\mathrm{p}<0.001]$. Peeling the ILM was likely to be cost-effective.

Conclusion: There was no evidence of a difference in distance VA following ILM peel and no-ILM peel techniques. An important benefit in favour of no ILM peeling was ruled out. Given the higher anatomical closure and lower re-operation rates in the ILM peel group, ILM peeling would seem the treatment of choice for idiopathic stage 2-3 FTMH.

(Clinical Trials.gov number, NCT00286507). 
An idiopathic full-thickness macular hole (FTMH) represents a defect in the area of maximal vision of the retina, the fovea; if left untreated often leads to severe central visual loss. ${ }^{1}$ FTMH are common, with an estimated incidence of 7.8 persons / 100,000 population per year. ${ }^{2}$ Macular hole surgery, first developed by Kelly and Wendell in 1991, ${ }^{3}$ represents one of the most common procedures performed by vitreo-retinal surgeons. ${ }^{4}$

Four stages (1-4) of FTMH have been described; ${ }^{5}$ randomized controlled clinical trials (RCTs) conducted in the 1990s showed that macular hole surgery was effective for stages 2, 3 and $4 .^{6,7}$

Peeling the internal limiting membrane (ILM) of the retina was introduced as an additional manoeuvre in macular hole surgery in order to improve anatomical and functional outcomes following surgery. ${ }^{8}$ Several observational studies suggested a benefit of peeling the ILM (reviewed by Abdelkader and Lois ${ }^{9}$ ). Furthermore, recent data from two small RCTs undertaken in China and Denmark, which included 49 and 75 patients, respectively, suggested a potential beneficial effect of this operation. ${ }^{10,11}$

The Full-thickness macular hole and Internal Limiting Membrane peeling Study (FILMS), herein reported, was designed as a “pragmatic” RCT to test the hypothesis that surgical peeling of the ILM for people with idiopathic FTMH (stages 2 or 3), compared with surgery without peel, improves vision, the likelihood of hole closure, participant reported health related quality of life and is cost-effective. "Pragmatic" RCTs aim to determine the effects of an intervention under the usual circumstances (i.e. normal clinical practice); as opposed to "explanatory" RCTs which are primarily designed to determine the effects of an intervention under ideal circumstances. ${ }^{12}$ The advantages of a pragmatic design are that if the treatment is effective it can be implemented into clinical practice.

\section{Methods}


The FILMS study was a multicenter, pragmatic RCT. Patients were enrolled at nine centres, seven in UK and two in the Republic of Ireland, by local clinical investigators. Eligible participants were those with stage 2 or 3 idiopathic FTMH of $\leq 18$ months duration and visual acuity (VA) $\leq 20 / 40$ in the study eye. At each participating centre, vitreo-retinal surgeons followed their usual clinical practice [including slit-lamp biomicroscopy, colour and red-free photographs, optical coherence tomography (OCT), fluorescein angiography and fundus autofluorescence (AF), as required] to stage FTMH and to confirm eligibility. Individuals with stage 1 or 4 FTMH, idiopathic FTMH stages 2-3 of $>18$ months duration, with other causes of decreased vision, and those with FTMH related to high myopia or trauma were excluded. Individuals that did not understand English and/or unable to give informed consent were also excluded. The Medical Research Council Good Clinical Practice Guidelines, 1998, and the UK Data Protection Act, 1998 were followed. Approval by the Multicentre Research Ethics Committee, Local Ethics Committees and local hospital trusts of each participating centre and participants’ written informed consent were obtained prior to the initiation of the study.

During the recruitment phase, two RCTs of ILM peeling versus no ILM peeling presented their results. ${ }^{10,11}$ In light of this, an independent Data Monitoring Committee was established (Jan 2007) and reviewed the external and accumulating FILMS trial evidence and recommended continuing recruitment.

Meetings, involving all individuals participating in this RCT (including vitreo-retinal surgeons, optometrists and photographers, in addition to those responsible for the running of the trial) were held prior to the initiation of the study to discuss the methodology of this RCT and issues regarding data collection.

\section{Study Treatment, randomisation and masking}


All participants received a combined phacoemulsification and pars plana vitrectomy, including detachment and removal of the posterior hyaloid, followed by a fluid-air exchange and air-gas [12\% perfluoropropane $\left.\left(\mathrm{C}_{3} \mathrm{~F}_{8}\right)\right]$ exchange. Participants were randomized 1:1 to the above procedure alone or combined with ILM peeling using a central telephone IVR system at the trial office (Centre for Healthcare Randomised Trials (CHaRT) - UK NIHR trials unit. In the latter, following ILM staining with trypan blue (0.15\% trypan blue under air for 3 minutes) removal of the ILM was performed in an area of around 1-2 disc diameters around the hole. Re-staining with trypan blue was permitted at the discretion of the vitreoretinal surgeon. Each vitreo-retinal surgeon judged whether the peeling of the ILM was “complete”, as defined by a removal of the ILM all around the hole within a minimal extension of 1 disk diameter centred on the centre of the hole or "incomplete" if otherwise. A minimisation algorithm (according to Taves, with $\mathrm{P}=1$ ) ${ }^{13}$ was used which allocated treatment with respect to minimising imbalance in trial centre, distance vision in the study and fellow eye (20/40-20/160; 20/200-20/500; <20/500), stage of the macular hole (2 or 3), duration of symptoms ( $\leq 1$ year; $>1$ year) and lens status (phakic, aphakic or pseudophakic). Participants were followed up at one, three and 6 months post-surgery.

Further surgical intervention was allowed in either group according to standard clinical practice if the macular hole remained opened following surgery, including ILM peeling in participants initially randomized to no peel. All participants were instructed to posture face down for a period of five-seven days post-surgery and received a posturing chart to record the time postured.

Participants and optometrists, who undertook the visual function evaluation of the participants, were masked to the treatment allocation. Vitreo-retinal surgeons, who were evaluating the status of the macula hole pre- and post-operatively and performing the surgery were, unmasked. 


\section{Outcomes}

The primary outcome was the mean difference between treatment groups in the Early Treatment Diabetic Retinopathy Study (ETDRS) distance VA score at six months. Secondary outcomes included ETDRS distance VA at three months, near VA (Bailey-Lovie) at three and six months, contrast sensitivity (Pelli-Robson) at six months, reading speed (MNRead) at six months, anatomical closure of the macular hole at each time point (one, three and six months), participant related outcomes, as determined by the EQ-5D and VFQ25 questionnaires, at six months, costs to the health service and the participant, incremental costs per quality adjusted life year (QALY) and adverse events.

The macular hole was considered to be closed when the hole was no longer visible clinically (open holes with or without subretinal fluid around them were considered to be open). At each participating centre, vitreo-retinal surgeons followed their usual clinical practice [including slit-lamp biomicroscopy, colour and red-free photographs, optical coherence tomography (OCT), fluorescein angiography and fundus autofluorescence (AF) as required] to determine whether the macular hole was open or closed.

\section{$\underline{\text { Sample size and statistical analysis }}$}

Assuming a common standard deviation of 12 ETDRS points in the two randomised groups, to detect a 6 ETDRS score difference (an effect size of 0.5) using a two sample two sided ttest at a $5 \%$ level of significance and $80 \%$ power, it was estimated that 64 participants would be required in each group. This calculation was based on data from published studies. ${ }^{14,15}$

The statistical analysis was based on the intention to treat principle: participants grouped as randomised irrespective of subsequent compliance. The primary outcome was compared between the two groups using a linear regression model adjusted for the baseline score and minimisation covariates. Statistical significance for the primary and secondary outcomes were based on two-sided tests with $2 \mathrm{P} \leq 0.05$ taken as the criterion for statistical 
significance. The principal analysis was based upon available case data with no imputation of missing values. Sensitivity analyses of the primary and macular hole closure outcomes which assessed the impact of missing data by imputing extreme values (lowest and highest/open and closed respectively) was also undertaken. Additionally, the primary outcome was analysed according to the pre-specified subgroups (lens status, duration and stage of the macular hole) by including the corresponding interaction term(s) in the regression model using stricter criteria for statistical significance $(2 \mathrm{P} \leq 0.01)$. Macular hole closure, complications and further surgery were analysed using logistic regression model with adjustment for the minimisation covariates (except study centre). Analyses of health related quality of life measures (EQ-5D and VFQ-25) and secondary measures of visual function (contrast sensitivity, reading speed and distance and near visual acuity) were undertaken using linear regression adjusted for baseline score in addition to the minimisation variables (bar centre). A mean difference was reported for a continuous outcome and an odds ratio (OR) for a binary outcome. The corresponding confidence interval (CI) and p-value were reported.

\section{$\underline{\text { Economic Evaluation }}$}

A cost per participant for each arm of the trial was calculated based upon the use of primary and secondary care services. Resource utilisation per participant was estimated using patient questionnaires and information recorded in the three and six month case report forms. Unit costs/prices were obtained using information from the National Health Service, published estimates for health care services and/or interventions, ${ }^{16-18}$ as well as study specific estimates. QALYs were calculated from responses to the EQ-5D using the area under the curve method with EQ-5D responses valued using UK population tariffs. ${ }^{19}$ Point estimates for mean costs and mean QALYs were derived for both ILM peel and no ILM peel groups. The mean difference in costs and QALY were estimated using analysis of covariance adjusting for 
minimisation factors and baseline EQ-5D for QALYS. These data were subsequently used to estimate an incremental cost per QALY gained.

The results were presented as point estimates of mean incremental costs, QALYS, and incremental cost per QALY. Measures of variance for these outcomes were estimated by bootstrapping costs, QALYs, and incremental cost per QALY. The incremental costeffectiveness data obtained by the bootstrapping exercise were presented in terms of costeffectiveness acceptability curves (CEACs).

Further details of the methodology used in FILMS have been published elsewhere. ${ }^{20}$ As stated in this published protocol, in addition to the above, in all participating centres colour photographs and red free images were obtained at each time point (baseline, 1, 3 and 6 months) and evaluated, in a masked fashion and using a computerised programme, to objectively determine the size of the hole prior to surgery and the status of the hole postoperatively. These data, not presented here, will form part of a separate study evaluating the effect of the size of the macular hole on outcomes following surgery. Furthermore, a longterm 24 month follow-up evaluation is also included.

\section{Results}

\section{$\underline{\text { Study Participants }}$}

Between July $1^{\text {st }} 2005$ and January $31^{\text {st }}$ 2009, 217 patients were approached; of these 76 were ineligible $(n=44)$ or declined $(n=32)$ taking part on the study. One-hundred and forty one were eligible, agreed to take part and were randomized (see CONSORT diagram, Figure 1). Of these, three were later identified as stage 4 FTMH and therefore were regarded as postrandomisation exclusions; these were not included in the analyses. Randomized treatment groups were generally well balanced with respect to baseline characteristics with the exception of gender and EQ-5D scores (Table 1). 


\section{$\underline{\text { Surgical details and intraoperative complications }}$}

Table 2 summarizes details of the surgery and intraoperative complications. More participants in the no ILM peel group had surgery performed under general anaesthesia. On average, the surgery took longer if the ILM was peeled than if it was not removed (72.6 versus 62.6 minutes). The ILM was completely removed in 64 of 67 participants receiving surgery in the ILM peel group; in three participants in this group the ILM was not removed; for one participant it was unknown whether they had surgery or not (Figure 1). Four participants in the no ILM peel group received ILM peeling and represented protocol deviations though retained in the analyses. No statistically significant differences in the number of intraoperative complications were found between groups [38 (57\%) versus 28 (42\%) OR: 1.73, 95\% CI (0.86, 3.50); $\mathrm{p}=0.166]$. For both groups, the most common intraoperative complication was the occurrence of retinal tears.

\section{Primary and Secondary Outcomes}

No statistically significant differences in distance visual acuity at six months (primary outcome) were detected between groups [4.8, 95\% CI (-0.3, 9.8); $\mathrm{p}=0.063]$ (Table 3). The result was not sensitive to missing data based upon the sensitivity analyses (results not shown). However, an adjusted mean difference of 5 ETDRS letters was observed in favour of the ILM peel group. None of the sub-group analyses of the primary outcome were found to be statistically significant (data not shown). No statistically significant differences were observed in other functional outcomes at any of the time points studied between groups (Table 3).

At the one month follow-up visit, macular hole closure was observed in 56 (84\%) participants in the ILM-peel group compared with 31 (48\%) in the no ILM peel group; this difference was statistically significant [OR: 6.23, 95\% CI $(2.01,19.30) ; \mathrm{p}<0.001]$. No 
statistically significant difference in rates of hole closure between ILM peel and no ILM peel groups was observed at three and six months. The sensitivity analysis results for macular hole closure did not change the above findings. A post-hoc subgroup analysis of macular hole closure by stage of the hole showed no significant difference [OR: 0.46, 95\% CI $(0.04,4.99)$; $\mathrm{p}=0.400]$. No statistically significant differences in responses to the EQ-5D and VFQ-25 scores were observed between groups.

\section{$\underline{\text { Postoperative complications }}$}

No statistically significant differences in the number of complications after initial surgery were found between groups [21 (31\%) versus 27 (41\%); OR: 0.63, 95\% CI $(0.31,1.31)$; $\mathrm{p}=$ 0.218]. Rhegmatogenous retinal detachment occurred in 5\% and 3\% of cases in ILM peel and no ILM peel groups, respectively. Post-operative complications have been summarized in Table 4. Eight (12\%) in the ILM peel group compared with 31 participants (48\%) in the no ILM peel group required at least one further surgery [OR: $0.14,95 \%$ CI $(0.05,0.34)$; $\mathrm{p}<0.001]$ (Table 4). This difference was driven by 25 (38\%) participants in the no ILM peel group undergoing peeling of the ILM within the follow-up period. There was one re-opening of the macular hole in the no ILM peel group and none in the ILM peel group.

\section{Economic evaluation}

The economic analysis revealed that the mean cost of care was $£ 2550$ in the ILM peel group and $£ 2974$ in the no ILM peel group [difference -£424, 95\% CI (-1045, £182)]. The main determinant of this difference in mean costs appeared to be the increased need for subsequent surgery in the no ILM peel group. The mean unadjusted QALY scores for each group were 0.413 in the ILM peel group and 0.438 in the no ILM peel group [difference $-0.025,95 \%$ CI (-0.424 to 0.452)]. The difference QALYS adjusted for baseline EQ-5D scores and other minimisation factors was 0.002 [95\% CI $(-0.01,0.013)]$. The difference in costs and effects 
was not statistically significant at the $5 \%$ level. However, based upon the bootstrapped estimates of cost-effectiveness it is probable that ILM peeling would be cost-effective at typical threshold values for society’s willingness to pay for a QALY (90\% chance of being considered cost-effective when society’s willingness to pay for a QALY is $£ 20,000$ ).

\section{Discussion}

FILMS found no evidence of a difference between groups in distance visual acuity. The observed difference, which favoured ILM peeling, was however of a clinically relevant magnitude (5 ETDRS letters). Furthermore, an important benefit in favour of no ILM peeling was ruled out. This, together with the higher rates of macular hole closure and corresponding fewer re-operations needed in the ILM peel group suggests that ILM peeling is the treatment of choice for patients with stage 2-3 FTMH.

Participants randomized to no-ILM peel received ILM peeling if the macular hole remained open; this most likely explains the lack of differences in macular hole closure rates at three and six months. The difference in the number of re-operations was the main driver of differences in cost between the two procedures. Indeed, the results suggest that there is over $90 \%$ chance that ILM peeling is less costly than the no peeling technique. Importantly, data from this study support a lack of a deleterious effect of ILM peeling in visual function, one of the main concerns raised of performing ILM peeling. ${ }^{21}$ The functional and anatomical outcomes achieved in FILMS are consistent with those from two other smaller trials comparing macular hole surgery with or without ILM peeling recently published. ${ }^{10,11}$ The benefit of ILM peeling has been also suggested by the results of one other RCT very recently completed, which included only participants $(n=80)$ with large holes (>400 microns) and which has been published only, to date, in an abstract form. ${ }^{22}$

Rates of primary hole closure (closure of the hole with a single surgery) of 84\% and 48\% achieved in the ILM peel and no ILM peel groups at one month, respectively, 
in FILMS may seem lower than those reported in previously published observational studies (summarized by Abdelkader and Lois). ${ }^{9}$ Case series, however, are highly prone to selection bias and reporting better outcomes than those seen in standard clinical practice. Most vitreoretinal surgeons (>80\%) participating in FILMS had extensive experienced in vitreo-retinal surgery, including macular hole surgery. Interestingly, anatomical results in FILMS are comparable to those obtained in one of the recently published RCTs with similar inclusion criteria, definition for hole closure and surgical protocol than FILMS, in which macular hole closure at three months was achieved in $44 \%$ and $89 \%$ in the no ILM peel group and in the trypan-blue assisted ILM group, respectively. ${ }^{10}$ Thus, it is likely that these lower rates of closure are representative of real clinical practice.

Cataract surgery was performed in FILMS at the time of the macular hole repair. This would have avoided progression of pre-existing cataracts to act as a possible confounder in the evaluation of the functional results. Furthermore, as complications reasonably accredited to the combined procedure seemed to be few and had no deleterious effects in vision (Table 5), phaco-vitrectomy for FTMH appears to be a reasonable and convenient option for patients and may be a more efficient use of resources.

FILMS represents the largest RCT conducted to date aimed to address the value of ILM peeling in surgery for idiopathic FTMH and the only one which has included an evaluation of the quality of life and cost-effectiveness of the interventions used. Although large for the field of Ophthalmology, and specifically, macular hole, this trial may not have had enough power to rule out a clinically significant difference in favour of peeling for the primary outcome. From a purely scientific viewpoint, the high rate of re-interventions and large proportion of participants in the no ILM peel group who subsequently received ILM peeling complicates the interpretation of the results. However, it is entirely consistent with clinical practice and naturally follows from the intention to treat philosophy as a comparison of two intervention pathways. 
FILMS randomised 141 participants (90\% completed the 6 month follow-up) in 9 centres in the UK and Eire. The study was intentionally designed as a pragmatic, as opposed to a more explanatory, trial thereby increasing the external validity (generalisability) of its findings by reflecting decision making in clinical practice. Vitreo-retinal surgeons determined the diagnosis and staging of FTMH and its status following surgery and the need for further surgery based on their own routine clinical practice. Participating surgeons used their own routine techniques to induce a posterior hyaloid detachment, to peel the ILM (with the exception of the use of trypan blue to stain the ILM) and to achieve an adequate gas exchange.

In conclusion, data from FILMS suggests that ILM peeling is a safe, clinically superior and more cost-effective approach than no ILM peeling to treat patients with idiopathic stage 2 or 3 FTMH and, thus, may be considered the treatment of choice for patients with this common vitreo-retinal disease.

\section{Acknowledgment}

We are indebted to all of the participants and to the Chief Scientist Office of the Scottish Government Health Directorates for funding the study. Jonathan Cook holds a Medical Research Council UK fellowship. The Health Services Research Unit and the Health Economics Research Unit are both core funded by the Chief Scientist Office of the Scottish Government Health Directorates. We thank the independent Data Monitoring Committee members: Professor Gordon Murray (chair), Mr Bill Aylward and Mr Tom Williamson. We wish to thank Mary Kilonzo and Robyn de Verteuil for their assistance in the economic evaluation of this trial, to Daniel Barnett for programming support and Diana Collins for data entry. 
Clinical investigators: Hatem Atta, Stephen Beatty, Catherine Cleary, Andrew Dick, John Ellis, John Forrester, Carl Groenewald, Richard Haynes, Henrich Heimann, Muhammad Irfan Khan, Dara Kilmartin, Noemi Lois, Asif Orakzai, CK Patel, Ian Pearce, Tarik Saddik, David Steel, David Wong. Optometrists and local co-ordinators: Charles Cottriall, Cherry Daly, Laura Duncan, Karon McEwing, Sarah Muir, Anita Murphy, Stan Keys, Lynda Lindsell and Valerie Tompkin. Photographers: Terri Ainley, Victor Beatty, Gillian Bennerson, Anne Bolton, Jon Brett, Alison Farrow, Ronnie Jackson, Tony Johnston, Marie Kinsella, Stephen Neilson, Hugh Nolan, Sarah Stanley and Jim Talbot. Medical imaging: Ayyakkawnu Manivannan. Data Monitoring Committee: Prof Gordon Murray (Chair), Mr Bill Aylward and Mr Tom Williamson.

\section{Authors' contributions}

NL conceived the study, participated in its design, coordination and interpretation of its results and drafted this manuscript. JB participated in the design, coordination and interpretation of its results and contributed to this manuscript. JN participated in the design of the study and its statistical plan and contributed to the manuscript. LV participated in the design, coordination and interpretation of its results, and contributed to this manuscript. JC participated in the design, drafted the statistical plan, supervised the analysis of the data, contributed to the interpretation of the results and contributed to the manuscript. CB undertook the statistical analysis of the data, contributed to the interpretation of the results and commented to the manuscript. AM participated in the design and coordination of the study and data management and contributed to the manuscript. LT undertook the economic evaluation of the data, participated on the interpretation of the results and contributed to the manuscript. Gladys McPherson participated on the coordination of the study and data management. All authors read and approved the final manuscript. 
ILM Peeling versus no peeling for idiopathic stage 2-3 FTMH/Lois et al.

NL, corresponding Author, has full access to all the data in the study and had final responsibility for the decision to submit the manuscript for publication. 


\section{References}

1. Casuso LA, Scott IU, et al. Long-term follow-up of unoperated macular holes.

Ophthalmology 2001;108:1150-5.

2. McCannel CA, Ensminger JL, Diehl NN, Hodge DN. Population-based incidence of macular holes. Ophthalmology 2009;116:1366-9.

3. Kelly NE, Wendel RT. Vitreous surgery for idiopathic macular holes. Results of a pilot study. Arch Ophthalmol 1991;109:654-9.

4. Ah-Fat FG, Sharma MC, Majid MA, McGalliard JN, Wong D. Trends in vitreoretinal surgery at a tertiary referral centre: 1987 to 1996. Br J Ophthalmol 1999, 83:385-6.

5. Gass JD. Idiopathic senile macular hole. Its early stages and pathogenesis. Arch Ophthalmol.1988;106:629-39.

6. Kim JW, Freeman WR, Azen SP, el-Haig W, Klein DJ, Bailey IL. Prospective randomized trial of vitrectomy or observation for stage 2 macular holes. Vitrectomy for Macular Hole Study Group. Am J Ophthalmol 1996;121:605-14.

7. Freeman WR, Azen SP, Kim JW, el-Haig W, Mishell DR III, Bailey I. Vitrectomy for the treatment of full-thickness stage 3 or 4 macular holes. Results of a multicentered randomized clinical trial. The Vitrectomy for Treatment of Macular Hole Study Group. Arch Ophthalmol 1997;115:11-21.

8. Eckardt C, Eckardt U, Groos S, Luciano L, Reale E. Removal of the internal limiting membrane in macular holes. Clinical and morphological findings. Ophthalmologe 1997;94:545-51. 
9. Abdelkader E, Lois N. Internal limiting membrane peeling in vitreo-retinal surgery. Surv Ophthalmol 2008;53:368-96.

10. Christensen UC, Kroyer K, Sander B, et al. Value of internal limiting membrane peeling in surgery for idiopathic macular hole stage 2 and 3: a randomised clinical trial. $\mathrm{Br} \mathrm{J}$

Ophthalmol 2009;93:1005-15.

11. Kwok AK, Lai TY, Wong VW. Idiopathic macular hole surgery in Chinese patients: a randomised study to compare indocyanine green-assisted internal limiting membrane peeling with no internal limiting membrane peeling. Hong Kong Med J 2005;11:259-66. 12. Thorpe KE, Zwarenstein M, Oxman AD, Treweek S, Furberg CD, Altman DG, Tunis S, Bergel E, Harvey I, Magid DJ, Chalkidou K. A pragmatic-explanatory continuum indicator summary (PRECIS): a tool to help trial designers. J Clin Epidemiol. 2009 May;62(5):464-75. 13. Taves DR. Minimization: A new method of assigning patients to treatment and control groups. Clinical pharmacology and therapeutics, 1974;15:443-453.

14. Brooks HL, Jr. Macular hole surgery with and without internal limiting membrane peeling. Ophthalmology. 2000;107:1939-48.

15. Paques M, Chastang C, Mathis A, et al. Effect of autologous platelet concentrate in surgery for idiopathic macular hole: results of a multicenter, double-masked, randomized trial. Platelets in Macular Hole Surgery Group. Ophthalmology 1999;106:932-8. 16. British National Formulary 57 [website on the Internet]. London: BMJ Group and RPS Publishing; 2009 [accessed June 2009]. Available from: URL:http://bnf.org/bnf/index.htm. 
17. ISD Scotland [database on the Internet]. Edinburgh: Information Services Division, NHS National Services Scotland; 2009 [accessed June 2009]. Available from: URL:

http://www.isdscotland.org/isd/1.html.

18. Curtis L Curtis L. Unit Costs for Health and Social Care [document on the Internet].

University of Kent: Personal Social Services Research Unit; 2008 [accessed June 2009].

Available from: URL: http://www.pssru.ac.uk/uc/uc2008contents.htm.

19. Dolan P, Gudex C, Kind P, et al. A social tariff for Euroqol: results from a UK general population survey. Discussion Paper 138 [document on the Internet]. University of York: Centre for Health Economics; 1995 [accessed June 2009]. Available form: URL: http://www.york.ac.uk/inst/che/pdf/DP138.pdf.

20. Lois N, Burr J, Norrie J, Vale L, Cook J, McDonald A, for the Full-thickness macular hole and internal limiting membrane peeling study (FILMS) Group. Clinical and costeffectiveness of internal limiting membrane peeling for patients with idiopathic full thickness macular hole. Protocol for a Randomised Controlled Trial: FILMS (Full-thickness macular hole and Internal Limiting Membrane peeling Study). Trials. 2008 Nov 3;9:61.

21. Terasaki H, Miyake Y, Nomura R, et al. Focal macular ERGs in eyes after removal of macular ILM during macular hole surgery. Invest Ophthalmol Vis Sci 2001;42:229-34. 22. Tadayoni R, Creuzot-Garcher C, Korobelnik JF, et al. Internal limiting membrane peeling for large macular holes: a randomized, multicentric, and controlled clinical trial. Invest Ophthalmol Vis Sci 2009;50:E-Abstract 5206. 
Figure 1: Consort diagram

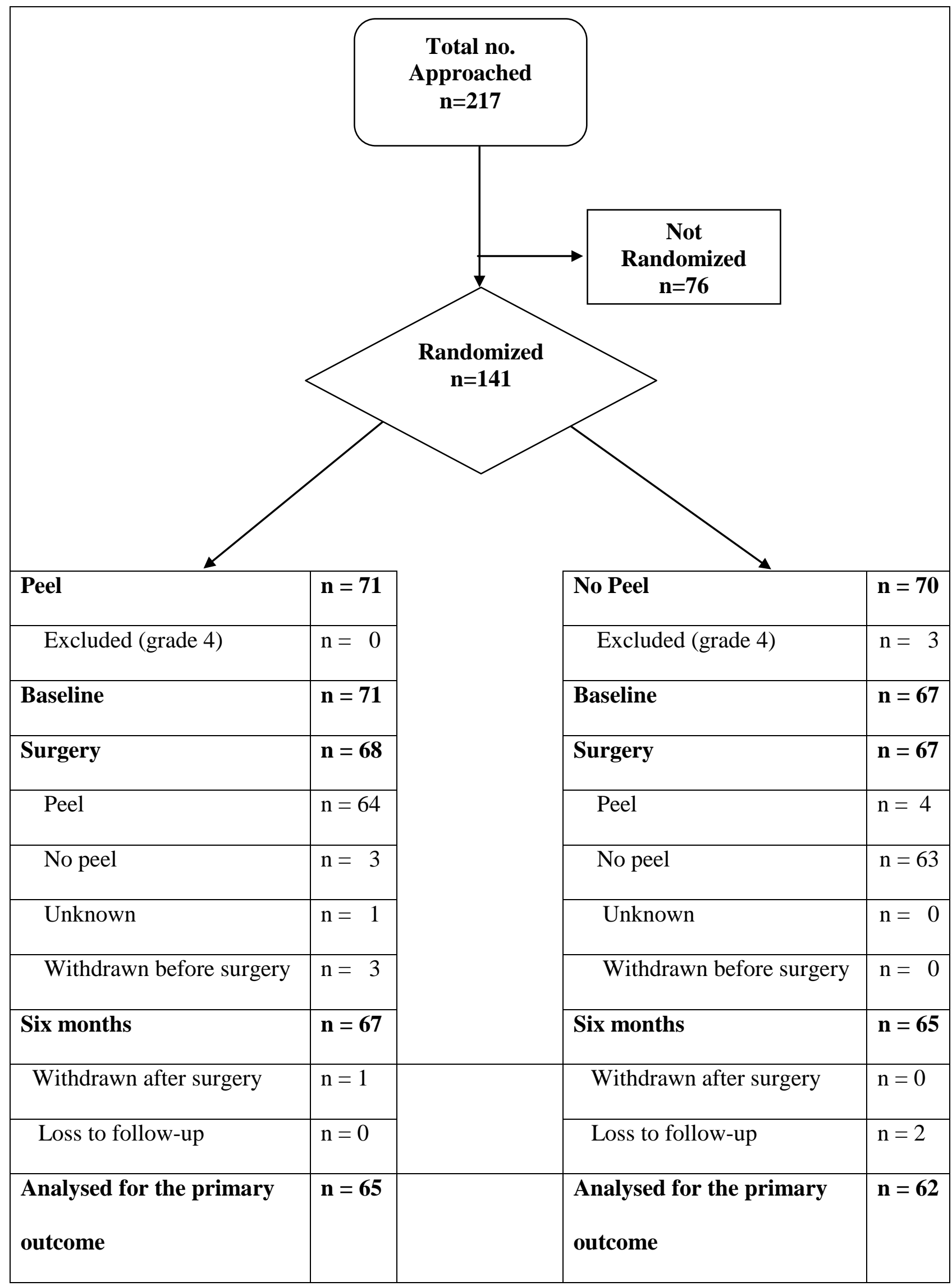


Table 1. Baseline characteristics

\begin{tabular}{|c|c|c|c|}
\hline Participant characteristic & & Peel & No peel \\
\hline \multirow[t]{2}{*}{ Study eye - n/N (\%) } & Right & $40 / 71(56)$ & $38 / 66(58)$ \\
\hline & Left & $31 / 71(44)$ & $28 / 66(42)$ \\
\hline Gender - n/N (\%) female & & $56 / 71(79)$ & $43 / 66(65)$ \\
\hline Age - Median (IQR) years & & $\begin{array}{l}70,70.30 \\
(5.72)\end{array}$ & $\begin{array}{c}66,70.58 \\
(6.02)\end{array}$ \\
\hline $\begin{array}{l}\text { Duration of symptoms - N, Mean } \\
\text { (SD) months }\end{array}$ & & $71,7.0(4.2)$ & $66,6.5(4.0)$ \\
\hline Status of lens $-\mathrm{n} / \mathrm{N}(\%)$ & $\begin{array}{l}\text { Phakic } \\
\text { Grade Nuclear Standard } \\
1 \text { to }<3 \\
3 \text { or more } \\
\text { Grade PSC Standard } \\
1 \text { to }<3 \\
3 \text { or more } \\
\text { Grade Cortical Standard } \\
<1 \\
1 \text { to }<3 \\
3 \text { or more }\end{array}$ & $\begin{array}{r}67 / 71(94) \\
20 / 64(31) \\
44 / 64(69) \\
0 / 64(0) \\
48 / 52(92) \\
4 / 52(8) \\
0 / 52(0) \\
34 / 53(64) \\
18 / 53(34) \\
1 / 53(2)\end{array}$ & $\begin{array}{r}64 / 67(96) \\
19 / 60(32) \\
41 / 60(68) \\
0 / 60(0) \\
43 / 50(86) \\
6 / 50(12) \\
1 / 50(2) \\
35 / 49(71) \\
14 / 49(29) \\
0 / 49(0)\end{array}$ \\
\hline
\end{tabular}


ILM Peeling versus no peeling for idiopathic stage 2-3 FTMH/Lois et al.

\begin{tabular}{|c|c|c|c|}
\hline & Pseudophakic & 4/71 (6) & 3/67 (4) \\
\hline \multirow{2}{*}{$\begin{array}{l}\text { Stage of macular hole - n/N (\%) } \\
\text { (Clinical classification) }\end{array}$} & II & 29/71 (41) & 25/66 (38) \\
\hline & III & 42/71 (59) & 41/66 (62) \\
\hline$E Q-5 D-N$, mean (SD) & & $\begin{array}{c}65, \\
0.80(0.21)\end{array}$ & $\begin{array}{c}63, \\
0.88(0.13)\end{array}$ \\
\hline VFQ-25 - N, mean (SD) & & $\begin{array}{c}66, \\
79.9(15.5)\end{array}$ & $\begin{array}{c}64, \\
80.3(17.5)\end{array}$ \\
\hline \multirow[t]{2}{*}{$\begin{array}{l}\text { Distance visual acuity (ETDRS) score } \\
-\mathrm{N} \text {, mean (SD) }\end{array}$} & Study eye & $\begin{array}{c}71, \\
48.1(13.8)\end{array}$ & $\begin{array}{c}66, \\
50.0(10.6)\end{array}$ \\
\hline & Fellow eye & $\begin{array}{c}71, \\
76.1(13.5)\end{array}$ & $\begin{array}{c}66, \\
75.7(17.1)\end{array}$ \\
\hline \multirow[t]{2}{*}{$\begin{array}{l}\text { Near visual acuity (Bailey-Lovie) - N, } \\
\text { mean (SD)) }\end{array}$} & Study eye & $\begin{array}{c}67, \\
0.86(0.30)\end{array}$ & $\begin{array}{c}65, \\
0.81(0.31)\end{array}$ \\
\hline & Fellow eye & $\begin{array}{c}67, \\
0.32(0.34)\end{array}$ & $\begin{array}{c}64, \\
0.27(0.32)\end{array}$ \\
\hline $\begin{array}{l}\text { Contrast Sensitivity (Pelli-Robson) - } \\
\text { N, mean (SD) }\end{array}$ & Study eye & $\begin{array}{c}66, \\
30.8(5.0)\end{array}$ & $\begin{array}{c}65, \\
30.8(5.0)\end{array}$ \\
\hline $\begin{array}{l}\text { Reading speed MN Read - N, mean } \\
\text { (SD) }\end{array}$ & Study eye & $\begin{array}{c}68, \\
137.76 \\
(55.7)\end{array}$ & $\begin{array}{c}64, \\
138.6(48.8)\end{array}$ \\
\hline
\end{tabular}


ILM Peeling versus no peeling for idiopathic stage 2-3 FTMH/Lois et al.

\begin{tabular}{|c|c|c|c|}
\hline & Binocular & 66, & 63, \\
& & $174.4(44.5)$ & $174.2(45.1)$ \\
\hline
\end{tabular}

$\mathrm{IQR}$ = interquartile range, PSC = posterior subcapsular cataract, $\mathrm{SD}=$ standard deviation, ETDRS = early treatment diabetic retinopathy study 
Table 2. Surgical details and intra-operative complications

\begin{tabular}{|c|c|c|c|}
\hline Surgical detail & Level & Peel & No peel \\
\hline \multirow[t]{2}{*}{ Surgeon grade $-\mathrm{n} / \mathrm{N}(\%)$} & Consultant & $55 / 67(82)$ & $51 / 65(78)$ \\
\hline & Fellow & 12/67 (18) & $14 / 65(22)$ \\
\hline \multirow[t]{6}{*}{ Assisting surgeon - n/N (\%) } & Consultant & $8 / 67(12)$ & 10/63 (16) \\
\hline & Fellow & 10/67 (15) & 10/63 (16) \\
\hline & Registrar & 25/67 (37) & $27 / 63(43)$ \\
\hline & $\mathrm{SHO}$ & $4 / 67(6)$ & $3 / 63(5)$ \\
\hline & Associate & $0 / 67(0)$ & $1 / 63(2)$ \\
\hline & None & $20 / 67(30)$ & 12/63 (19) \\
\hline \multirow[t]{2}{*}{ Type of anaesthesia $-\mathrm{n} / \mathrm{N}(\%)$} & General & $28 / 67(42)$ & 35/65 (54) \\
\hline & Local & 39/67 (58) & $30 / 65(46)$ \\
\hline $\begin{array}{l}\text { Operation time }-\mathrm{N} \text {, mean } \\
\text { (SD) }\end{array}$ & Minutes & $\begin{array}{c}62, \\
72.6(19.0)\end{array}$ & $\begin{array}{c}63, \\
62.6(21.7)\end{array}$ \\
\hline ILM peeling - n/N (\%) & & 64/67 (96) & 4/67 (6\%) \\
\hline $\begin{array}{l}\text { Any intraoperative } \\
\text { complication* - n/N (\%) }\end{array}$ & & 38/67 (57) & 28/65 (43) \\
\hline Retinal tears $-\mathrm{n} / \mathrm{N}(\%)$ & & 18/67 (27) & 21/65 (32) \\
\hline $\begin{array}{l}\text { Retinal detachment }-\mathrm{n} / \mathrm{N} \\
(\%)\end{array}$ & & $4 / 67(6)$ & $1 / 65(2)$ \\
\hline $\begin{array}{l}\text { Retinal haemorrhages }-\mathrm{n} / \mathrm{N} \\
\text { (\%) }\end{array}$ & & 13/67 (19) & $4 / 65(6)$ \\
\hline
\end{tabular}


ILM Peeling versus no peeling for idiopathic stage 2-3 FTMH/Lois et al.

\begin{tabular}{|l|l|c|c|}
\hline Retinal whitening - n/N (\%) & & $9 / 67(13)$ & $1 / 65(2)$ \\
\hline Choroidal/Suprachoroidal & & $2 / 67(3)$ & $0 / 65(0)$ \\
\hline Other & & & \\
\hline
\end{tabular}

*Some participants had more than one complication 
Table 3. Primary and secondary outcomes

\begin{tabular}{|c|c|c|c|}
\hline Primary outcome & Peel & No peel & $\begin{array}{l}\text { Mean diff (95\% CI); p- } \\
\text { value }\end{array}$ \\
\hline $\begin{array}{l}\text { Distance visual acuity at six } \\
\text { months; N, mean (SD) }\end{array}$ & $\begin{array}{c}65, \\
61.3(14.7)\end{array}$ & $\begin{array}{c}62, \\
59.2(18.9)\end{array}$ & 4.8 (-0.3 to 9.8$) ; 0.063$ \\
\hline Secondary visual outcomes & Peel & No peel & $\begin{array}{l}\text { Mean diff (95\% CI); p- } \\
\text { value }\end{array}$ \\
\hline $\begin{array}{l}\text { Distance visual acuity at three } \\
\text { months; N, mean (SD) }\end{array}$ & $\begin{array}{c}66, \\
59.1(15.4)\end{array}$ & $\begin{array}{c}65, \\
56.7(19.7)\end{array}$ & 3.6 (-1.9 to 9.1$) ; 0.201$ \\
\hline $\begin{array}{l}\text { Near visual acuity at three } \\
\text { months; N, mean (SD) }\end{array}$ & $\begin{array}{c}65, \\
0.62(0.32)\end{array}$ & $\begin{array}{c}60, \\
0.63(0.38)\end{array}$ & $-0.04(-0.15,0.06) ; 0.406$ \\
\hline $\begin{array}{l}\text { Near visual acuity at six } \\
\text { months; N, mean (SD) }\end{array}$ & $\begin{array}{c}62, \\
0.54(0.31)\end{array}$ & $\begin{array}{c}61, \\
0.53(0.31)\end{array}$ & $-0.03(-0.12,0.07) ; 0.559$ \\
\hline $\begin{array}{l}\text { Contrast sensitivity at six } \\
\text { months; N, mean (SD) }\end{array}$ & $\begin{array}{c}63, \\
31.4(4.8)\end{array}$ & $\begin{array}{c}60, \\
32.1(4.2)\end{array}$ & $-0.7(-2.0,0.7) ; 0.332$ \\
\hline $\begin{array}{l}\text { Reading speed (study eye) at six } \\
\text { months; N, mean (SD) }\end{array}$ & $\begin{array}{c}61, \\
168(53)\end{array}$ & $\begin{array}{c}59, \\
157(47)\end{array}$ & $11(-3,26) ; 0.123$ \\
\hline $\begin{array}{l}\text { Reading speed (binocular) at six } \\
\text { months; N, mean (SD) }\end{array}$ & $\begin{array}{c}60, \\
188(44)\end{array}$ & $\begin{array}{c}59, \\
182(46)\end{array}$ & $4(-7,14) ; 0.498$ \\
\hline Secondary outcomes: QOL & Peel & No peel & $\begin{array}{l}\text { Mean diff (95\% CI); p- } \\
\text { value }\end{array}$ \\
\hline EQ-5D six months; $N$, mean & 53 & 59 & $0.02(-0.05,0.08) ; 0.600$ \\
\hline
\end{tabular}


ILM Peeling versus no peeling for idiopathic stage 2-3 FTMH/Lois et al.

\begin{tabular}{|c|c|c|c|}
\hline (SD) & $0.85(0.20)$ & $0.87(0.15)$ & \\
\hline $\begin{array}{l}\text { VFQ-25 six months; N, mean } \\
\text { (SD) }\end{array}$ & $\begin{array}{c}55, \\
85.7(11.6)\end{array}$ & $\begin{array}{c}59, \\
85.8(13.2)\end{array}$ & $0.4(-3.3,4.1) ; 0.847$ \\
\hline $\begin{array}{l}\text { Secondary outcome: macular } \\
\text { hole status - n/N (\%) closed }\end{array}$ & Peel & No peel & $\begin{array}{l}\text { Odds ratio (95\% CI);p- } \\
\text { value }\end{array}$ \\
\hline one month & $56 / 67(84)$ & $31 / 64(48)$ & $\begin{array}{l}6.23(2.64 \text { to } 14.73) ; \\
<0.001\end{array}$ \\
\hline three months & $61 / 66(92)$ & $52 / 63(83)$ & 2.65 (0.84 to 8.36); 0.097 \\
\hline six months & $61 / 65(94)$ & 56/63 (89) & 1.92 (0.52 to 7.11$) ; 0.330$ \\
\hline
\end{tabular}


Table 4. Complications and further eye surgery reported during the follow-up (up to six months)

\begin{tabular}{|c|c|c|c|}
\hline Event - n/N (\%) & Peel & No peel & $\begin{array}{l}\text { Odds ratio (95\% } \\
\text { CI); p-value }\end{array}$ \\
\hline Any complication* & 21/64 (31) & 27/62 (44) & \multirow{7}{*}{$\begin{array}{l}0.63(0.31,1.31) ; \\
0.218\end{array}$} \\
\hline Anterior uveitis & $1 / 64(2)$ & $1 / 62(2)$ & \\
\hline Decentration of the IOL & $0 / 64(0)$ & $3 / 62(5)$ & \\
\hline Increased IOP & 6/64 (9) & 9/62 (15) & \\
\hline Retinal detachment & 3/64 (5) & 2/62 (3) & \\
\hline RPE changes at macula & 7/64 (11) & 9/62 (15) & \\
\hline Other & 8/64 (13) & 6/62 (11) & \\
\hline Any further eye surgery* & 8/67 (12) & 31/65 (48) & \multirow{8}{*}{$\begin{array}{l}0.14(0.05,0.34) ; \\
<0.001\end{array}$} \\
\hline ILM peel & 2/67 (3) & 25/65 (38) & \\
\hline Surgery for retinal detachment & 2/67 (3) & 2/65 (3) & \\
\hline Redo macular hole & 2/67 (3) & 5/65 (8) & \\
\hline Vitrectomy + gas & 2/67 (3) & $0 / 65(0)$ & \\
\hline Yag capsulotomy & $0 / 67(0)$ & $1 / 65(2)$ & \\
\hline Removal of oil & $1 / 67(1)$ & 0/65 (0) & \\
\hline Epiretinal membrane peel & 1/67 (1) & $0 / 65(0)$ & \\
\hline
\end{tabular}

* Some participants had more than one complication or further surgery 